Supporting information for

\title{
Localized Photothermal Ablation Therapy of Obstructive Rectal Cancer using a Nanofunctionalized Stent in a Mouse Model
}

Hong-Tao $\mathrm{Hu}^{1,2 \dagger}$, Jung-Hoon Park ${ }^{3 \dagger}$, Zhe Wang ${ }^{4 \dagger}$, Nader Bakheet ${ }^{5}$, Shi-Jun $\mathrm{Xu}^{1,2}$, Eunji Lee ${ }^{1}$, Dong-Hyun $\mathrm{Kim}^{6}$, Song Hee Kim ${ }^{3}$, Ho-Young Song 7 , Jae Yong Jeon ${ }^{8 *}$, Suhwan Chang ${ }^{1 *}$

${ }^{[\dagger]}$ H.-T.H, J.-H.P. and Z.W. contributed equally to this work and are the co-first authors.

${ }^{1}$ Department of Biomedical Sciences, Asan Medical Center, University of Ulsan College of Medicine, 88 Olympic-ro 43-gil, Songpa-gu, Seoul, 05505, Republic of Korea.

${ }^{2}$ Department of Minimal-Invasive Intervention, The Affiliated Cancer Hospital of Zhengzhou University, 127 Dongming Road, Zhengzhou 450003, Henan Province, China.

${ }^{3}$ Biomedical Engineering Research Center, Asan Institute for Life Sciences, Asan Medical Center, 88 Olympic-ro 43-gil, Songpa-gu, Seoul, 05505, Republic of Korea

${ }^{4}$ Department of Radiology, Tianjin Medical University General Hospital, Anshan Road 154\#, Heping District, CN 300052, China.

${ }^{5}$ Gastrointestinal Endoscopy and Liver unit, Kasr Al-Ainy, Faculty of Medicine, Cairo University, Cairo, Egypt.

${ }^{6}$ Department of Radiology, Feinberg School of Medicine, and Robert H. Lurie Comprehensive Cancer Center, Northwestern University, Chicago, IL, 60611, USA

${ }^{7}$ Department of Radiology, UT Health Science Center at San Antonio, 7703 Floyd Curl Drive, San Antonio, TX, 78229, USA

${ }^{8}$ Department of Rehabilitation, Asan Medical Center, University of Ulsan College of Medicine, 88 Olympic-ro 43-gil, Songpa-gu, Seoul, 05505, Republic of Korea.

\section{Corresponding Authors:}

Jae Yong Jeon, M.D., Ph.D.

Department of Rehabilitation, Asan Medical Center, University of Ulsan College of Medicine

88 Olympic-ro 43-gil, Songpa-gu, Seoul 05505, Republic of Korea

Tel: 82-2-3010-3791 Fax: 82-2-476-0090

E-mail: jyjeon71@gmail.com

Suhwan Chang, Ph.D.

Department of Biomedical Sciences, Asan Medical Center, University of Ulsan College of Medicine

88 Olympic-ro 43-gil, Songpa-gu, Seoul 05505, Republic of Korea

Tel: 82-2-3010-2095 Fax: 82-2-476-0090

E-mail: suhwan.chang@amc.seoul.kr 


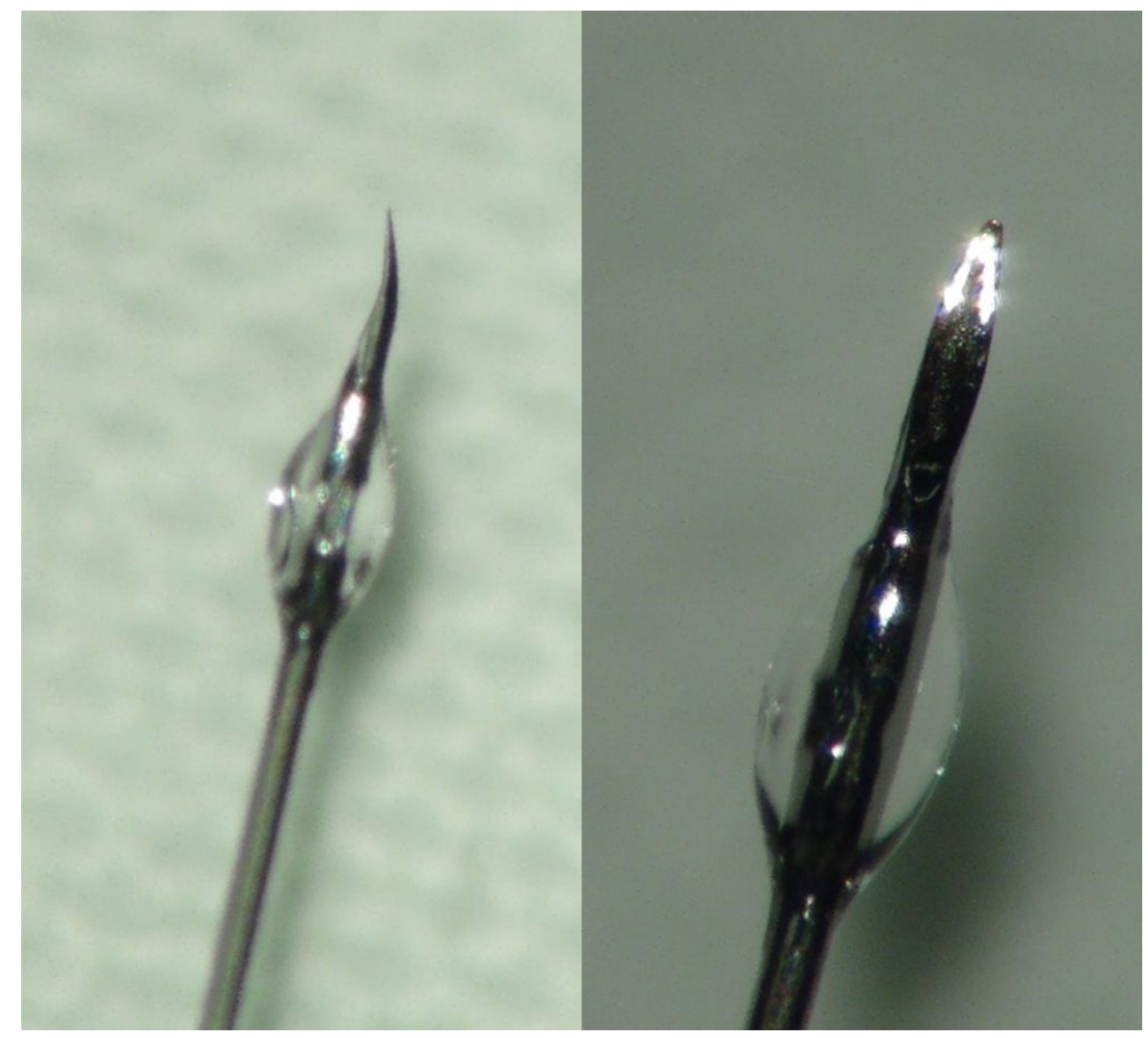

Supplementary Figure 1. Customized $30 \mathrm{G}$ needle $\left(15 \mathrm{~cm}\right.$ length, $45^{\circ}$ bevel of the needle tip) for tumor cell implantation into the colonic sub-mucosa and bulb formation. 


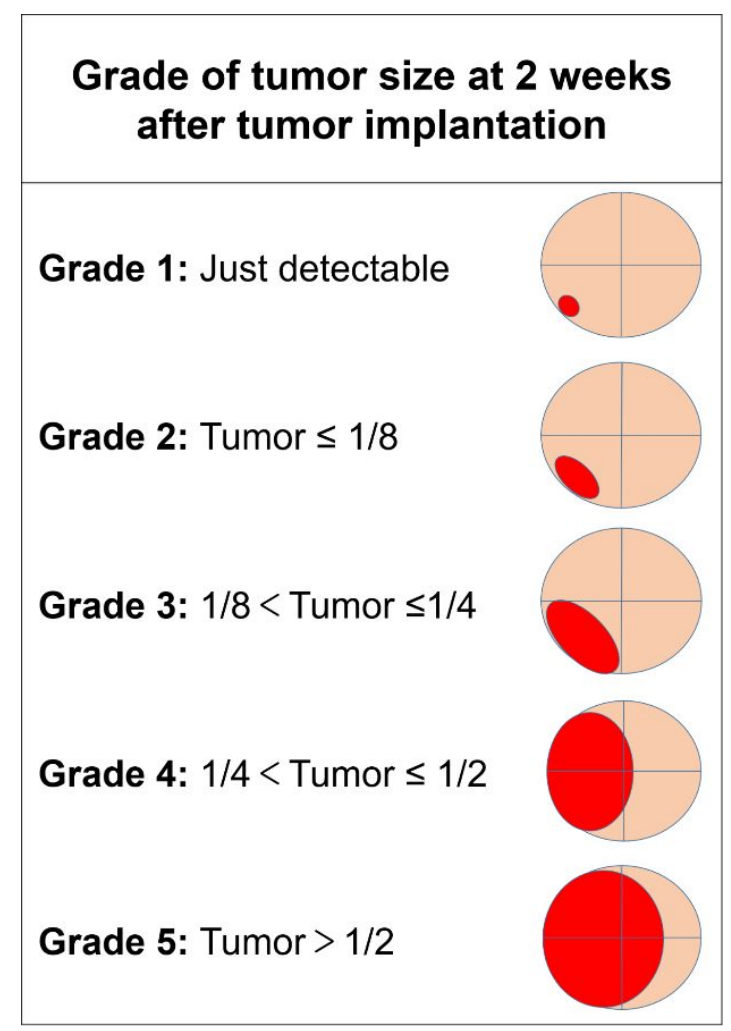

Supplementary Figure 2. The grading score of tumor size relative to the circumference of the mouse colon. 


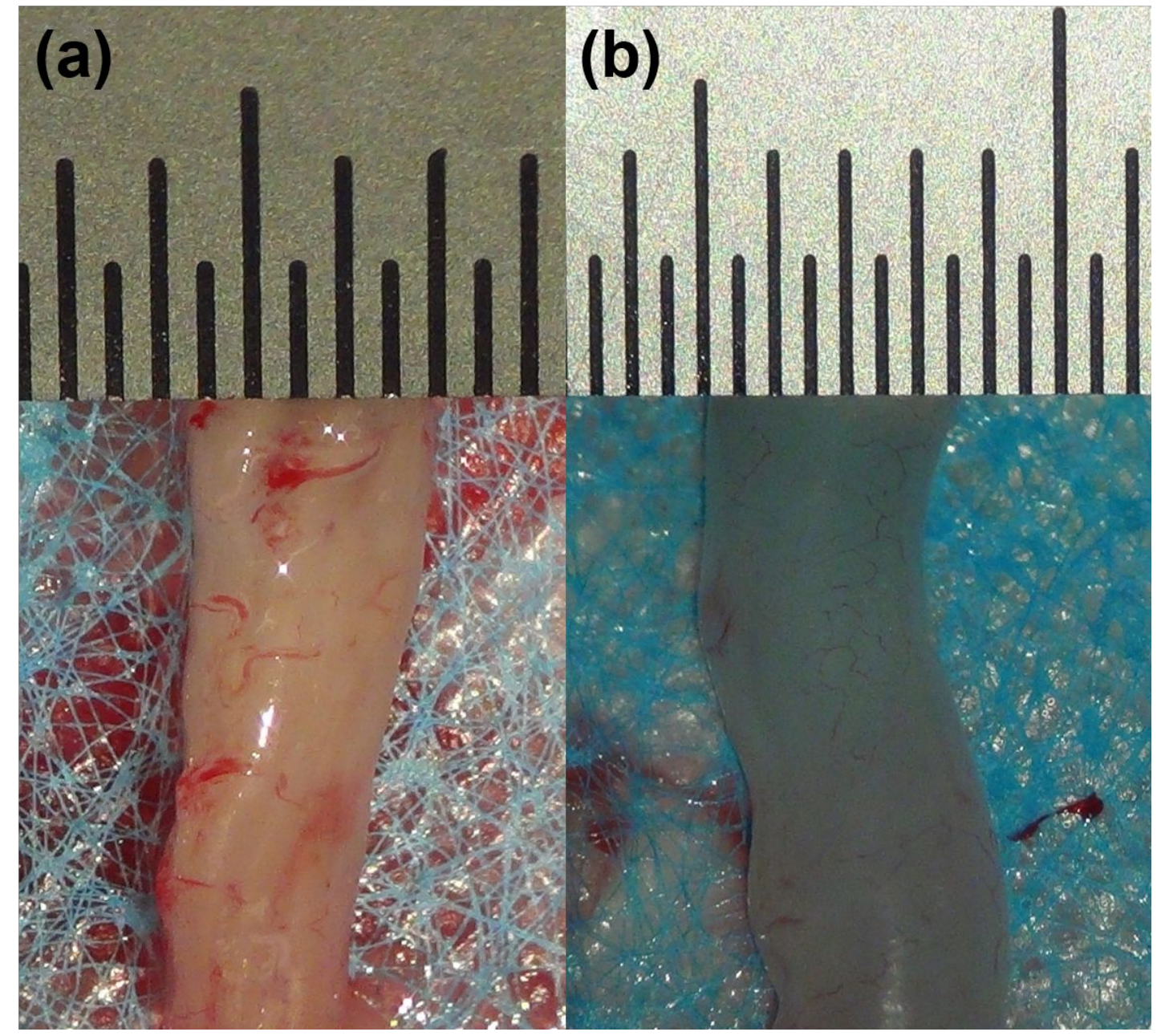

Supplementary figure 3. Ex vivo colon diameter measurement. The diameter of the normal colon and the water-filled colon were measured by a ruler. For the normal situation colon, the diameter is about $2.5 \mathrm{~mm}$. But for the water-filled colon, the colon diameter almost achieved $3.5 \mathrm{~mm}$ ( $8 \mathrm{x}$ magnification). 


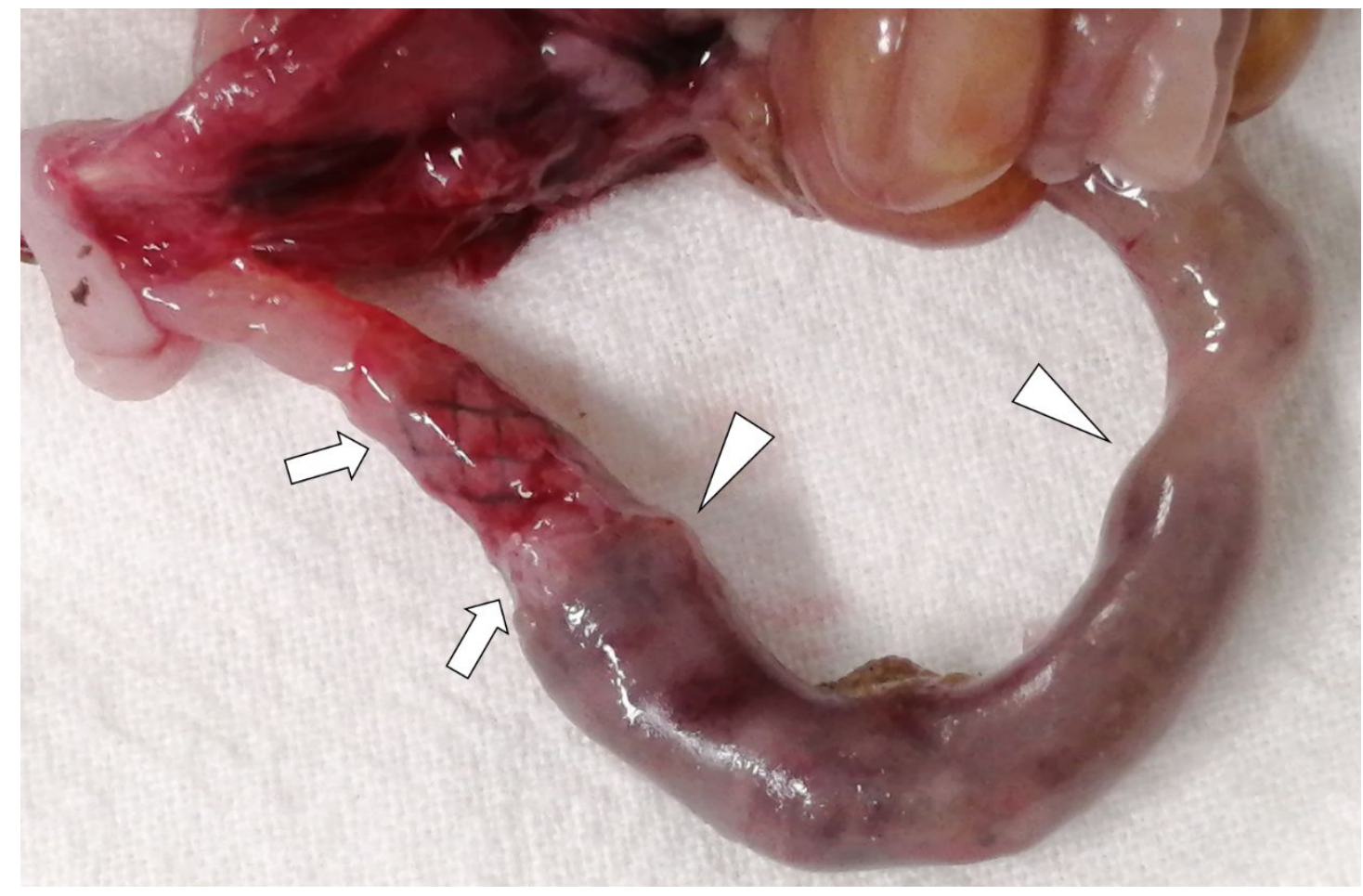

Supplementary Figure 4. Entire colon extracted from a mouse with colonic obstruction caused by stool impaction (arrowheads) after stent placement (arrows). 